\title{
BMJ open Cohort study of the association of hypnotic use with mortality in postmenopausal women
}

\author{
Arthur Hartz, John Jacob Ross
}

To cite: Hartz A, Ross JJ. Cohort study of the association of hypnotic use with mortality in postmenopausal women. BMJ Open 2012;2:e001413. doi:10.1136/bmjopen-2012001413

\section{- Prepublication history and} additional material for this paper are available online. To view these files please visit the journal online (http://dx. doi.org/10.1136/bmjopen2012-001413).

Received 2 May 2012 Accepted 20 July 2012

This final article is available for use under the terms of the Creative Commons Attribution Non-Commercial 2.0 Licence; see http://bmjopen.bmj.com

Health Services Research, Huntsman Cancer Institute, Salt Lake City, Utah, USA

Correspondence to Dr Arthur Hartz; hartzarthur@gmail.com

\begin{abstract}
Objective: Previous studies found an association between hypnotic use and mortality risk. The prospective outcome data and the many baseline risk factors included in the Women's Health Initiative (WHI) provide an opportunity to better understand the reasons for this association.
\end{abstract}

Setting: The WHI is a long-term national health study that focused on strategies for preventing disease in postmenopausal women. Participants were enrolled from 1993 to 1998.

Design: Baseline hypnotic use was evaluated for an association with subsequent mortality or disease after adjusting for baseline risk.

Subjects: 148938 postmenopausal women between the ages of 50 and 79 throughout the USA. The median follow-up was 8 years.

Main outcome measures: Mortality. Secondary outcomes included myocardial infarction, stroke, diabetes and seven types of cancer.

Results: For persons who use hypnotic medications almost daily the age-adjusted hazard ratio (HR) for mortality was 1.62 (95\% Cl 1.50 to 1.74). Greater hypnotic use was associated with less healthy levels of physical function, general health and smoking at baseline. After adjustment for these factors the HR for almost daily hypnotic use was 1.14 (1.06 to 1.23 ) for mortality and 1.53 (1.18 to 1.99) for melanoma; it was not significantly associated with increased incidence of other diseases tested. Less frequent hypnotic use and several types of sleeping difficulties were not associated with mortality, but sleeping more than $10 \mathrm{~h}$ a night had a risk-adjusted HR for mortality of 1.28 (1.01 to 1.61 ).

Conclusions: The association of hypnotic use with mortality and incident disease was greatly reduced after adjusting for baseline risk factors. These findings do not support a strong independent association of hypnotic use with most health outcomes.

Inadequate sleep is a predictor of numerous health problems including depression, ${ }^{1}$ hypertension, ${ }^{2}$ diabetes ${ }^{3}$ and cardiac events. ${ }^{4}$ To reduce these problems many people rely on hypnotics. Americans filled some 60 million prescriptions for hypnotics last year, up from

\section{ARTICLE SUMMARY}

\section{Article focus}

- The study evaluated whether hypnotic use at baseline was associated with subsequent mortality or disease incidence. The analysis took into account baseline health.

\section{Key messages}

- The results supported previous findings of an association of hypnotic use with subsequent poor health or mortality. However, this association was almost eliminated after taking into account smoking, general health and physical function at baseline. These risk factors may have exaggerated the apparent health consequences of hypnotic use in other studies.

Strengths and limitations of this study

- Strengths include a large data set with information about hypnotic use, sleep disturbance and risk factors for mortality. A limitation is that many currently used hypnotics were not available at the time of this study.

47 million in 2006, according to IMS Health, a healthcare services company. ${ }^{5}$ Despite their obvious acute effects, their long-term efficacy for treatment of sleep disorders is unclear. ${ }^{6-8}$ In addition, there are concerns that these medications may have adverse health effects. A recent study found high hazard ratios for mortality and cancer associated with hypnotic use. ${ }^{5} 910$ Other studies have also found evidence that hypnotic use may increase the risk of disease and mortality. ${ }^{11-19}$

A potential flaw in these studies, however, is the possibility that higher-risk patients take more hypnotics, and it is this higher risk rather than the hypnotics that contributes to adverse health outcomes. For example, it might be that health problems or psychological problems interfere with sleep, and people with these problems are more likely to take sleeping pills. It is also possible that sleep problems rather than sleep medications cause adverse health outcomes. 
Although observational studies do not dependably produce valid results ${ }^{20}$ a number of features of the Women's Health Initiative (WHI) dataset make it better than most for evaluating the influence of sleep medication on health. It includes a large and, in many respects, diverse population with accurately recorded health outcomes that occur during a median follow-up time of 8 years. It also provides baseline information about characteristics of sleep, use of sleep medication and numerous risk factors. In the present study WHI data were analysed to assess the association of sleep medication, hours sleep and difficulty sleeping with mortality and the development of 10 specific diseases.

\section{METHODS}

The WHI study design has been described in detail. ${ }^{21-23}$ In brief, it was a long-term national health study that focused on strategies for preventing heart disease, breast and colorectal cancer, and osteoporosis in postmenopausal women. Women between the ages of 50 and 79 were enrolled for an observational study or randomised controlled trial (RCT) from 1993 to 1998 at 40 clinical centres throughout the USA. The institutional review boards at all participating institutions, including the coordinating centre, subcontractors and clinical centres, approved the study protocols and procedures.

Participants available for analysis included 161748 WHI participants: 93 651from the observational study, 16590 from the RCT of oestrogen plus progesterone $(\mathrm{E}+\mathrm{P}), 10722$ from the RCT of oestrogen only (oestrogen-alone), and 40785 additional women who were in the diet study and not in an RCT of hormone therapy. Baseline information included demographics, general health, clinical and anthropometric, functional status, healthcare behaviours, reproductive, medical history, family history, personal habits, thoughts and feelings, therapeutic class of medication, hormones, supplements and dietary intake. Outcomes were identified primarily through self-report at semiannual contacts for clinical trial participants and annual contacts for observational study participants. Specific details of illnesses and hospitalisations are obtained as needed via a standardised questionnaire administered by phone or in-person interview, or self-completed form. For primary and secondary outcomes, portions of the medical record (discharge summary and results of relevant diagnostic and laboratory tests) are requested and assembled.

We excluded 9584 participants from the observational study because they would have been excluded from RCTs. Exclusion criteria were platelets less than $75000 / \mathrm{mm}^{3}$, haematocrit less than $32 \%$, oral daily use of a glucocorticosteroid, body mass index (BMI) less than 18, systolic blood pressure greater than $200 \mathrm{~mm} \mathrm{Hg}$, diastolic greater than $105 \mathrm{~mm} \mathrm{Hg}$, breast cancer ever, other cancers in the last 10 years, or stroke, transient ischaemic attack, or myocardial infarction (MI) in the last 6 months. These exclusion criteria made the study participants more homogenous (since they had already been applied to the RCTs) and they reduced the likelihood that sleep patterns would be influenced by severe health problems. An additional 3285 participants were excluded from the final analyses because they were missing information on one or more of the questions used to define difficulty sleeping. The number of participants analysed was 148938 .

\section{Outcomes and risk factors}

The present study assessed the association of hypnotic use with mortality (the primary outcome in this study and the one most often evaluated for an association with sleep medications) and other disease outcomes that may contribute to mortality: MI, stroke, diabetes, breast cancer, colon cancer, lung cancer, colorectal cancer, lymphoma, melanoma and ovarian cancer. If hypnotic use caused specific health problems that led to mortality, then the strongest associations should be between sleep medication use and these health problems. It is certainly possible that the health problems most associated with hypnotic use were not collected by the WHI or tested in the present study. One of the outcomes cited in several studies of hypnotic use that was not collected by the WHI was suicide. ${ }^{17} 1824$

Several risk factors related to sleep were tested for an association with the primary and secondary outcome variables.

The sleep factor analysed in greatest detail was the response to the following question about sleep medications: 'Did you take any kind of medication or alcohol at bedtime to help you sleep?' The answers were on a fivepoint scale from not in the past 4 weeks to 5 or more times a week. For simplicity the highest point on the scale was referred to as frequent hypnotic use.

Information about sleep duration was from the response to the following question: "About how many hours of sleep did you get on a typical night during the past 4 weeks?' Answers were on a six-point scale that ranged from 5 or less hours to 10 or more hours.

Information was also obtained about the following insomnia-related factors in the past 4 weeks: quality of sleep, trouble falling asleep, wake up several times, wake up earlier than planned, trouble getting back to sleep and the WHI sleep construct that was formed by summing the scores for the five specific types of difficulty sleeping.

These risk factors were tested for an association with the primary and secondary outcomes after adjusting for a set of risk factors highly associated with mortality as described in statistical methods. One of the mortality risk factors was smoking history, which was obtained from three questions:

1. A three category smoking status variable (never/ past/current).

2. On the average, how many cigarettes do you (did you) usually smoke each day?

3. How many years have you been (were you) a regular smoker? 


\section{Statistical methods}

The association of risk factors with each outcome was tested using Cox proportional hazard regression. Each of the risk factors related to sleep were evaluated separately. The hazard ratio (HR) and associated $\chi^{2}$ of the risk factor were obtained after adjusting for age and again after adjusting for mortality risk factors. The mortality risk factors were identified using stepwise proportional hazards regression on all the potential risk factors collected at baseline by the WHI. Factors statistically significant at $\mathrm{p}<0.0001$ level in the full dataset minus participants who would not qualify for RCTs were retained in the model.

The sleep variables were included in the regression models as categorical variables. The category of greatest interest for each sleep variable was the most extreme. Therefore, results were provided for the sleep variable as a whole and for the most extreme category of the sleep variable, which was represented by a binary variable.

The three questions about smoking were included as categorical risk factors in the proportional hazard regression equation. A composite smoking variable for a given subject was created by summing the age-adjusted regression coefficients associated with the values of the smoking variable present in that subject. This composite was an ordinal variable that was associated with an identical $\chi^{2}$ value as the $\chi^{2}$ is associated with the three categorical smoking variables. Because the variable was ordinal, it was possible to find its correlation with other risk factors.

The question about sleep medication was worded so that it included subjects who used alcohol to help them sleep. To assess whether the results were altered by subjects using alcohol as a sleep aid, the analyses on sleep medications were repeated for only those subjects who took less than one drink a week.

Every study that has evaluated the association between use of sleep medications and mortality adjusted for different covariables. The covariables that most influence the association between sleep medication use and mortality are those most confounded with sleep medications. The amount of confounding depends on what other variables are included in the regression equation. We created a score that measures the extent that frequent hypnotic use was confounded with another risk factor that was included in the Cox model. This score is analogous to a measure of confounding in linear regression. ${ }^{25}$ The confounding score was the product of $\chi_{\mathrm{j}}$ and $\mathrm{r}_{\mathrm{hu}, \mathrm{j}}$ where $\chi_{j}$ is the square root of the $\chi^{2}$ value for the other risk factor when it is in the Cox model with frequent hypnotic use, and $r_{h u, j}$ is the correlation of the other risk factor with frequent hypnotic use. This score provides an intuitive, quantified measure of the confounding associated with a particular covariable in the present study. For the 10 binary or ordinal covariables in the present study, the correlation of this confounding score with the change in the $\chi^{2}$ value of frequent hypnotic use when the covariable was added to the regression equation was 0.99 .

To test whether hypnotic use was a special risk factor for the morbidly obese, as has been suggested, ${ }^{26}$ we used a statistical test for interaction between hypnotic use and BMI of 35 or greater.

\section{RESULTS}

General characteristics of the participants are shown in table 1. For all variables except for race there is substantial diversity among the participants.

The risk factors independently associated with mortality at $\mathrm{p}<0.0001$ level are sorted in table 2 according to their $\chi^{2}$ value. The last column shows the score suggesting how much a variable is confounded with frequent hypnotic use. Three covariables have much higher confounding scores than the others: physical functioning, smoking and general health. Although age is by far the strongest risk factor, it is not highly confounded with the use of sleep medication.

The associations of mortality with characteristics of sleep are shown in table 3. All associations are shown adjusted for age alone, age plus the characteristics most confounded with frequent hypnotic use, and all covariables statistically significant at the $\mathrm{p}<0.0001$ level. The $\chi^{2}$ value for the association of hypnotic use with mortality adjusted for age is 182. After also adjusting for three confounding covariables, the $\chi^{2}$ value for sleep medications was reduced from 182 to 21 , and the HR for hypnotic use five or more times a week compared to non-users was reduced from $1.62(95 \% \mathrm{CI}=1.50$ to 1.74$)$ to 1.14 (1.06 to 1.23$)$. The risk-adjusted HRs for mortality associated with hypnotic use less frequent than five times a week were 1 or less.

The $\chi^{2}$ values were much greater for hypnotic use than for the other sleep characteristics. Of the six

\begin{tabular}{lll} 
Table 1 Subject characteristics & \\
\hline & & $\begin{array}{l}\text { Percentage } \\
\text { (N=148 938) }\end{array}$ \\
Characteristics & Categories & 17.8 \\
Age (years) & $49-55$ & 60.9 \\
& $56-69$ & 21.3 \\
Race & $70-81$ & 82.7 \\
Marital status & White & 62.4 \\
Education level & High-school graduate & 22.3 \\
& or less & \\
& Post high school & 38.5 \\
& College graduate or & 39.1 \\
Income & higher & \\
& $<U S \$ 35000$ & 42 \\
& US $\$ 35000$ to US $\$ 75000$ & 37.9 \\
Health score & $\geq U S \$ 75000$ & 17.4 \\
& $<75$ & 41 \\
& $75-90$ & 43 \\
& $>90$ & 9 \\
\hline
\end{tabular}


Table 2 Risk factors independently associated with mortality at $p<0.0001$ and the amount of confounding of these risk factors with frequent hypnotic use

\begin{tabular}{llccc}
\hline Risk factor & HR & $\chi^{2}$ & $\begin{array}{c}\text { Correlation with } \\
\text { hypnotic use }\end{array}$ & $\begin{array}{c}\text { Confounding score } \\
\text { for hypnotic use }\end{array}$ \\
\hline Age (10 years) & 2.31 & 2343.5 & 0.007 & 0.44 \\
Smoking* $_{\text {Diabetes treatment }}$ & 1.38 & 1539.8 & 0.050 & 2.17 \\
Physical function* & 1.99 & 357.1 & 0.021 & 0.73 \\
General health & 0.82 & 332.5 & -0.137 & 7.48 \\
Coronary bypass surgery & 0.99 & 112.0 & -0.134 & 5.15 \\
Systolic blood pressure & 1.92 & 102.6 & 0.014 & 0.33 \\
Income* & 1.12 & 60.3 & 0.014 & 0.39 \\
Loss of appetite & 0.92 & 48.6 & -0.036 & 0.68 \\
Alcohol * & 1.19 & 48.6 & 0.052 & 0.98 \\
WHI dataset (3 df) & 0.94 & 25.6 & -0.001 & 0.01 \\
US region (3 df) & & 51.6 & & \\
\hline
\end{tabular}

*The HR was computed for an increase in the variable of $1 \mathrm{SD}$.

HR, hazard ratio; 3df, a variable with four categories has three degrees of freedom in the Cox model; WHI, Women's Health Initiative.

Table 3 Sleep factors associated with mortality

\begin{tabular}{|c|c|c|c|c|c|c|c|}
\hline \multirow[b]{3}{*}{ Parameter } & \multirow[b]{3}{*}{ Sample size } & \multicolumn{6}{|c|}{ Adjustment } \\
\hline & & \multicolumn{2}{|c|}{ Age only } & \multicolumn{2}{|c|}{$\begin{array}{l}\text { Age }+3 \text { primary } \\
\text { confounders }\end{array}$} & \multicolumn{2}{|c|}{$\begin{array}{l}10 \text { Variable } \\
\text { model }\end{array}$} \\
\hline & & HR & $\chi^{2^{*}}$ & $\overline{\text { HR }}$ & $\chi^{2^{*}}$ & $\overline{\text { HR }}$ & $\chi^{2^{\star}}$ \\
\hline \multicolumn{8}{|l|}{ Sleep meds } \\
\hline $5+/$ week & 9142 & 1.62 & 156.8 & 1.14 & 11.2 & 1.14 & 10.9 \\
\hline 3-4/week & 4187 & 1.34 & 24.0 & 1.04 & 0.5 & 1.06 & 1.0 \\
\hline $1-2 /$ week & 8247 & 1.11 & 5.1 & 0.95 & 1.3 & 0.97 & 0.5 \\
\hline$<1 /$ week & 13737 & 0.96 & 1.3 & 0.91 & 5.6 & 0.94 & 2.2 \\
\hline Never & 113625 & 1.00 & $\mathrm{~N} / \mathrm{A}$ & 1.00 & $\mathrm{~N} / \mathrm{A}$ & 1 & $\mathrm{~N} / \mathrm{A}$ \\
\hline Overall & & & 181.8 & & 20.81 & & 16.0 \\
\hline \multicolumn{8}{|l|}{ Trouble initiating sleep } \\
\hline $5+$ times a week & 5864 & 1.54 & 81.5 & 1.05 & 1.1 & 1.02 & 0.1 \\
\hline 3-4 times a week & 8635 & 1.18 & 13.4 & 0.93 & 2.5 & 0.93 & 2.9 \\
\hline 1-2 times a week & 19105 & 1.05 & 2.5 & 0.92 & 6.3 & 0.93 & 4.8 \\
\hline$<1$ time a week & 27583 & 0.93 & 5.6 & 0.9 & 12.5 & 0.91 & 10.5 \\
\hline Never & 87751 & 1.00 & $\mathrm{~N} / \mathrm{A}$ & 1.00 & $\mathrm{~N} / \mathrm{A}$ & 1 & $\mathrm{~N} / \mathrm{A}$ \\
\hline Overall & & & 107.2 & & 20.1 & & 15.0 \\
\hline \multicolumn{8}{|l|}{ Quality of sleep } \\
\hline Very restless & 3293 & 1.49 & 28.2 & 0.86 & 3.9 & 0.84 & 5.4 \\
\hline Restless & 20914 & 1.25 & 27.1 & 0.87 & 10.1 & 0.89 & 7.0 \\
\hline Average quality & 62492 & 1.07 & 3.5 & 0.89 & 10.5 & 0.91 & 7.4 \\
\hline Sound or restful & 42936 & 1.01 & 0.0961 & 0.96 & 1.2 & 0.98 & 0.4 \\
\hline Very sound or restful & 19303 & 1.00 & $\mathrm{~N} / \mathrm{A}$ & 1.00 & $\mathrm{~N} / \mathrm{A}$ & 1 & $\mathrm{~N} / \mathrm{A}$ \\
\hline Overall & & & 64.4 & & 18.5 & & 16.3 \\
\hline \multicolumn{8}{|l|}{ Hours of sleep per night } \\
\hline 5 or less & 12517 & 1.38 & 68.5 & 1.1 & $5.4 \dagger$ & 1.05 & 1.3 \\
\hline 6 & 40966 & 1.14 & 22.4 & 1.06 & 4.3 & 1.04 & 2.1 \\
\hline 7 (control) & 55953 & 1.00 & $N / A$ & 1.00 & $\mathrm{~N} / \mathrm{A}$ & 1 & $\mathrm{~N} / \mathrm{A}$ \\
\hline 8 & 33041 & 1.06 & 3.1 & 1.04 & 1.9 & 1.04 & 1.9 \\
\hline 9 & 5695 & 1.23 & 13.8 & 1.08 & 1.8 & 1.06 & 1.1 \\
\hline 10 or more & 766 & 2.04 & 37.0 & 1.35 & $6.4 \dagger$ & 1.28 & 4.3 \\
\hline Overall & & & 109.1 & & 12.6 & & 6.8 \\
\hline
\end{tabular}

*If $\chi^{2} \geq 3.84, p<0.05$. If $\chi^{2} \geq 14.4, p<0.0001$.

†After adjusting for the other six risk factors for mortality, the $\chi^{2}$ value was reduced to 1.3 for sleeping $5 \mathrm{~h}$ or less and reduced to 4.3 for sleeping $10 \mathrm{~h}$ or more.

$\mathrm{N} / \mathrm{A}$, not applicable. 
Table 4 Association of sleep medication use with outcomes other than mortality

\begin{tabular}{|c|c|c|c|c|c|c|c|c|}
\hline \multirow[b]{3}{*}{ Outcome } & \multicolumn{4}{|c|}{ Age adjusted } & \multicolumn{4}{|c|}{ Adjusted for age +3 primary confounders } \\
\hline & \multicolumn{4}{|c|}{ Hypnotic use 5+/week } & \multicolumn{4}{|c|}{ Hypnotic use 5+/week } \\
\hline & HR & $\chi^{2 \star \star}$ & $\mathrm{CI}$ & & HR & $\chi^{2 \star \star}$ & $\mathrm{Cl}$ & \\
\hline Myocardial infarction & 1.35 & 21.7 & 1.19 & 1.53 & 1.02 & 0.05 & 0.89 & 1.15 \\
\hline Stroke & 1.35 & 18.1 & 1.17 & 1.54 & 1.09 & 1.5 & 0.95 & 1.25 \\
\hline Diabetes & 1.17 & 14.9 & 1.08 & 1.27 & 0.85 & 15.4 & 0.78 & 0.92 \\
\hline Breast cancer & 1.11 & 3.7 & 1.00 & 1.22 & 1.12 & 4.7 & 1.01 & 1.24 \\
\hline Colon cancer & 0.70 & 6.4 & 0.53 & 0.92 & 0.64 & 9.7 & 0.49 & 0.85 \\
\hline Lung cancer & 1.58 & 20.6 & 1.30 & 1.92 & 1.17 & 2.3 & 0.96 & 1.43 \\
\hline Colorectal cancer & 0.71 & 6.8 & 0.55 & 0.92 & 0.66 & 10.2 & 0.51 & 0.85 \\
\hline Lymphoma & 1.09 & 0.3 & 0.79 & 1.52 & 1.04 & 0.05 & 0.74 & 1.45 \\
\hline Melanoma & 1.35 & 5.2 & 1.04 & 1.74 & 1.53 & 10.4 & 1.18 & 1.99 \\
\hline Ovary cancer & 0.94 & 0.1 & 0.65 & 1.36 & 0.93 & 0.1 & 0.64 & 1.36 \\
\hline
\end{tabular}

measures of difficulty sleeping, only two were included in this table. The three other measures of specific types of difficulty sleeping had lower age-adjusted HRs and lower $\chi^{2}$ values than the reported measures.

The only other sleep-related variable associated with a statistically significant increased mortality risk was sleeping more than $10 \mathrm{~h}$ a night compared to sleeping $7 \mathrm{~h} \mathrm{a}$ night; the fully adjusted HR was 1.28 (1.01 to 1.61 ). Very short sleepers had a $33 \%$ age-adjusted elevation in mortality that was nearly eliminated with full adjustment for mortality risk factors. Perhaps some mortality risk factors contributed to very short sleep. Neither quality of sleep, trouble falling asleep, waking up several times, waking up earlier than planned, trouble getting back to sleep, nor the WHI sleep construct was significantly associated with increased mortality after adjusting for the three primary confounders.

There were statistically significant associations between frequent hypnotic use and difficulties sleeping although the correlations were perhaps lower than expected: 0.20 for difficulty initiating sleep, -0.12 for quality of sleep and -0.01 with number of hours of sleep.

It is possible that some participants in this study used alcohol as their sleep medication. After eliminating participants who used alcohol at least once a week, the risk-adjusted HR for using sleep medications at least five times a week compared to non-users of hypnotics was 1.13, $\mathrm{p}=0.01$. This HR is almost identical to the HR of 1.14 for hypnotic use at least five times a week in table 3 . HRs for other frequencies of hypnotic use after eliminating those who drank alcohol infrequently were all less than 1 and not statistically significant.

We found that frequent hypnotic use was a slightly stronger risk factor for women with BMI less than 35 than for more obese women, but the difference was not statistically significant, that is, the $\mathrm{p}$ value for the interaction was greater than 0.10 .

The association of hypnotic use with other outcomes is shown in table 4. Hypnotic use at least five times a week has age-adjusted statistically significant HRs for lung cancer, myocardial infarction, stroke and melanoma. After adjusting for the most highly confounded variables, the only HR significantly greater than 1 is for melanoma.

\section{DISCUSSION}

Our results supported previous findings of an association of the use of sleep medicine with incident disease and mortality. The associations were greatly reduced when adjustment was made for baseline physical function, health and smoking history. After the additional adjustment there was evidence that persons who used sleep medications almost daily had a slightly elevated risk of mortality and a substantially elevated risk of melanoma; there was no evidence that less frequent users had any additional risk of mortality or of relatively common specific diseases that might mediate the association between hypnotic use and mortality. Frequent hypnotic use had no stronger association with mortality for morbidly obese women. We also did not find evidence that difficulty sleeping was associated with mortality after adjusting for confounders. There was, however, a risk-adjusted association between sleeping more than $10 \mathrm{~h}$ a night and mortality.

A hypothesis consistent with these results is that sleep medications contributed little to greater mortality or disease but that they were used more frequently by patients at higher risk of mortality or disease. The association of mortality with more than $10 \mathrm{~h}$ a night of sleep might be eliminated by adjustment for additional baseline risk factors that contributed to mortality risk as well as long sleep.

\section{Previous literature}

Several previous studies found a statistically significant association between sleep medication and mortality ${ }^{9} 11-18$ although some did not. ${ }^{24} 2728$ The highest HRs were 5.3 for greater than 132 pills a year and 3.6 for the use of only 4-18 pills a year. ${ }^{9}$ Other HRs for regular users were between 2.03 (in a study with only 39 regular users) ${ }^{17}$ and 
1.09. ${ }^{27}$ The highest HRs for non-regular users ranged from $4.43^{9}$ to $0.98 .^{13}$

The studies differed with respect to setting (Sweden, ${ }^{17}$ Norway, ${ }^{12}$ Finland, ${ }^{13}$ the USA, ${ }^{9} 152728$ Canada, ${ }^{11} 18$ France $^{24}$ and Japan ${ }^{14}$ ) and conditions being treated by hypnotics (difficulty sleeping only ${ }^{9} 14-18242728$ or difficulty sleeping or anxiety ${ }^{11-13}$ ), and both of these differences influence the type of people who choose to take hypnotics and therefore the mortality risk factors that are confounded with hypnotic use. For example, it is possible that in some cultures a higher percentage of people who use hypnotics are depressed and therefore at an increased risk for suicide.

Only one study used data from a RCT, ${ }^{15}$ and the others differed greatly with respect to the number and types of risk factors used for adjustment, which impacts the ability of the study to remove confounding. No previous studies included information about any of the three variables most confounded with sleep medication use in the present study: physical function (which may be more relevant than information about exercise), precise smoking history and general health. It is possible that adjusting for these risk factors in previous studies would have weakened the risk found in these studies. It is also possible that study results may depend on the specific hypnotics used, which were rarely specified but no doubt varied substantially depending on the date of the study and the country in which the study was done.

Our study did find that the risk-adjusted HR for melanoma was 1.53 for frequent hypnotic use. This is consistent with a significant association of hypnotic use with skin cancer in a secondary analysis of RCT data ${ }^{15}$ and a laboratory study showing that these drugs cause cancer in animals. ${ }^{15}$ Other studies have also previously found an association between hypnotic use and cancer. ${ }^{19}$

\section{CONCLUSIONS}

Results from this study are consistent with many interpretations including a weak causal association of hypnotics with mortality, an association with mortality only because hypnotic use is a marker for poor health, and a strong association with mortality for recent hypnotics that were not well represented in this study. This study did not support a strong association of the hypnotics most commonly used in the 1990s with mortality. One possible reason that the results differed from some previous studies is that the most important confounding variables in the present study may not have been available in previous studies.

Acknowledgements The Women's Health Initiative Study (WHI) is conducted and supported by the NHLBI in collaboration with the WHI Investigators. This manuscript was prepared using a limited access dataset obtained by the $\mathrm{NHLBI}$ and does not necessarily reflect the opinions or views of the WHI or the NHLBI. The research was supported in part by the Huntsman Cancer Foundation and the Beaumont Foundation.
Contributors AH designed the study, directed the analyses and prepared the manuscript. JJR programmed the statistical packages and made suggestions for the analyses and manuscript revisions.

\section{Competing interests None.}

Ethics approval The institutional review boards at all participating institutions, including the coordinating centre, subcontractors and clinical centres, approved the study protocols and procedures.

\section{Patient consent Obtained.}

Provenance and peer review Not commissioned; externally peer reviewed.

Data sharing statement Investigators can apply to obtain the data used for this study from NHLB.

\section{REFERENCES}

1. Breslau N, Roth T, Rosenthal L, et al. Sleep disturbance and psychiatric disorders: a longitudinal epidemiological study of young adults. Biol Psychiatry 1996;39:411-18.

2. Suka M, Yoshida K, Sugimori H. Persistent insomnia is a predictor of hypertension in Japanese male workers. J Occup Health 2003;45:344-50.

3. Nilsson PM, Roost M, Engstrom G, et al. Incidence of diabetes in middle-aged men is related to sleep disturbances. Diabetes Care 2004;27:2464-9.

4. Asplund R. Sleep and cardiac diseases amongst elderly people. J Intern Med 1994;236:65-71.

5. Rabin RC. New worries about sleeping pills. Well: New York Times, 2012.

6. Morin CM, Vallières A, Guay B, et al. Cognitive behavioral therapy, singly and combined with medication, for persistent insomnia. JAMA 2009;301:2005-15.

7. Curran HV, Collins R, Fletcher S, et al. Older adults and withdrawal from benzodiazepine hypnotics in general practice: effects on cognitive function, sleep, mood and quality of life. Psychol Med 2003;33:1223-37.

8. Ohayon MM, Caulet M, Arbus L, et al. Are prescribed medications effective in the treatment of insomnia complaints? J Psychosom Res 1999;47:359-68.

9. Kripke DF, Langer RD, Kline LE. Hypnotics' association with mortality or cancer: a matched cohort study. BMJ Open 2012;2: e000850.

10. Greene G. The case for sleep medicine. New York Times, 2012 SR14.

11. Belleville G. Mortality hazard associated with anxiolytic and hypnotic drug use in the National Population Health Survey. CanJ Psychiatry 2010;55:558-67.

12. Hausken AM, Skurtveit S, Tverdal A. Use of anxiolytic or hypnotic drugs and total mortality in a general middle-aged population. Pharmacoepidemio Drug Saf 2007;16:913-18.

13. Hublin C, Partinen M, Koskenvuo M, et al. Sleep and mortality: a population-based 22-year follow-up study. Sleep 2007;30:1245-53

14. Kojima M, Wakai K, Kawamura T, et al. Sleep patterns and total mortality: a 12-year follow-up study in Japan. J Epidemiol/Jap Epidemiol Assoc 2000;10:87-93.

15. Kripke DF. Possibility that certain hypnotics might cause cancer in skin. J Sleep Res 2008;17:245-50.

16. Kripke DF, Garfinkel L, Wingard DL, et al. Mortality associated with sleep duration and insomnia. Arc Gen Psychiatry 2002;59:131-6.

17. Mallon L, Broman JE, Hetta J. Is usage of hypnotics associated with mortality? Sleep Med 2009;10:279-86.

18. Neutel $\mathrm{Cl}$, Patten SB. Risk of suicide attempts after benzodiazepine and/or antidepressant use. Ann Epidemiol 1997;7:568-74.

19. Kao CH, Sun LM, Liang JA, et al. Relationship of zolpidem and cancer risk: a Taiwanese population-based cohort study. Mayo Clin Proc. Mayo Clinic 2012;87:430-6.

20. Taubes G. Do we really know what makes us healthy? New York Times 2007

21. The Women's Health Initiative Study Group. Design of the Women's Health Initiative clinical trial and observational study. Control Clin Trials 1998;19:61-109.

22. Anderson GL, Manson J, Wallace R, et al. Implementation of the Women's Health Initiative study design. Ann Epidemiol 2003; 13(9 Suppl):S5-17.

23. Curb JD, McTiernan A, Heckbert SR, et al. Outcomes ascertainment and adjudication methods in the Women's Health Initiative. Ann Epidemiol 2003;13(9 Suppl):S122-8. 
24. Rod $\mathrm{NH}$, Vahtera J, Westerlund $\mathrm{H}$, et al. Sleep disturbances and cause-specific mortality: results from the GAZEL cohort study. Am J Epidemiol 2011;173:300-9.

25. Hartz A, Houts $P$, Arnold $S$, et al. A method to quantify confounding in regression analyses applied to data on diet and CHD incidence. J Clin Epidemiol 1988:41:331-7.
26. Vozoris NT, Leung RS. Sedative medication use: prevalence, risk factors, and associations with body mass index using population-level data. Sleep 2011;34:869-74.

27. Kripke DF, Klauber MR, Wingard DL, et al. Mortality hazard associated with prescription hypnotics. Biol Psychiatry 1998;43:687-93.

28. Phillips B, Mannino DM. Does insomnia kill? Sleep 2005;28:965-71. 